\title{
¿Es esto Educación Física?: el tiempo de compromiso motor en Educación Primaria
}

Is this Physical Education?: the motor commitment in Elementary Education

Adrián López-Taveras; Irene Moya-Mata

Facultad de Educación. Universidad de Valencia

Contacto: adlota041@gmail.com 


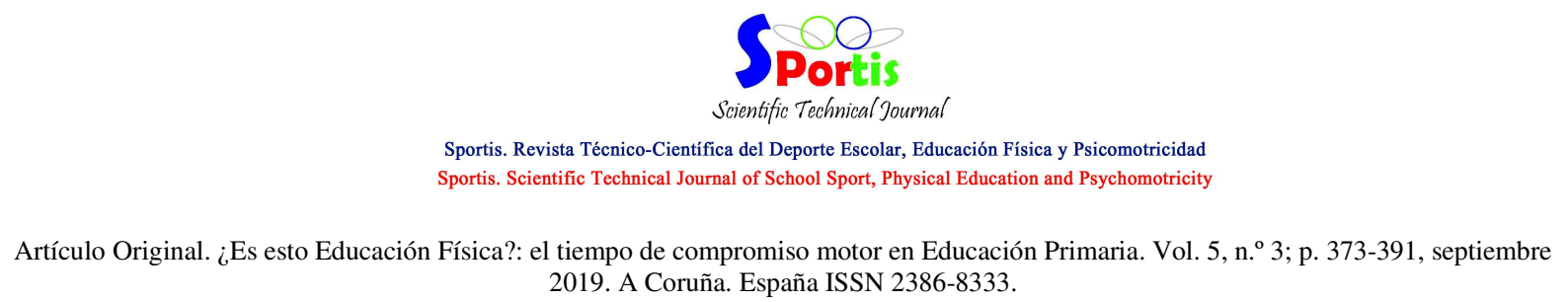
2019. A Coruña. España ISSN 2386-8333.

\title{
Resumen
}

La Educación Física ha de promover el desarrollo de hábitos de vida saludables y la prevención de enfermedades como la obesidad infantil. Para ello es necesario conocer el tiempo que el alumnado está en movimiento realizando actividad física en nuestras clases. Por lo tanto, el presente estudio tiene como objetivo analizar el tiempo de compromiso motor (TCM) en las sesiones de Educación Física (EF) en la etapa de Educación Primaria (EP). La muestra estuvo formada por 38 participantes de $1^{\circ}$ y $5^{\circ}$ curso. En total, se registraron nueve sesiones de EF impartidas por un único maestro especialista mediante una hoja de registro elaborada ad hoc. Los resultados muestran bajos valores de TCM en ambos cursos, siendo la media en primer curso de un $28,53 \%$ y en quinto curso de un $27,05 \%$. Por lo que el TCM en el primer curso es mayor que en quinto. Lo que supone que algo más del $70 \%$ del tiempo de sesión no se destina a la práctica motriz. Es necesario que los docentes de EF reflexionen no sólo sobre la cantidad de tiempo en las sesiones de EF, sino también en la calidad de éste, tratando de aumentar el TCM a través de tareas específicas que encaminen al alumnado a mejorar los hábitos de vida saludables.

\section{Palabras clave}

Educación Física; análisis observacional; tiempo de práctica; gestión del tiempo de clase.

\begin{abstract}
Physical Education must promote the development of healthy living habits and the prevention of diseases such as childhood obesity. For this reason it's necessary to know the time that student is in movement doing physical activity in our classes. Therefore, the present study aims to analyse the motor commitment time (MCT) in the Physical Education (PE) sessions in the Primary Education (PrE). The sample consisted of 38 pupils, 1st and 5th grade. In all, nine sessions of PE taught by a specialist teacher was recorded using an ad hoc record sheet. The results show low MCT values in both courses, being the average in the first course of $28.53 \%$ and in the fifth grade of $27.05 \%$. So the MCT in first grade is greater than in fifth. Wich implies that something more than $70 \%$ of the session time is not intended for driving practise. PE teachers need to reflect not only on the amount of time in PE sessions, but also on their quality, trying to increase the MCT througth specific tasks that will help students to improve healthy living habits.
\end{abstract}

\section{Keywords}

Physical Education; observational analysis; practice time; class time management.

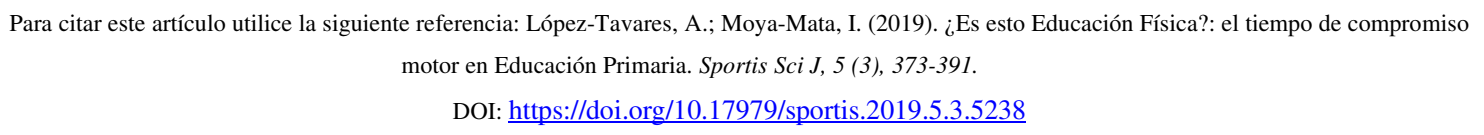




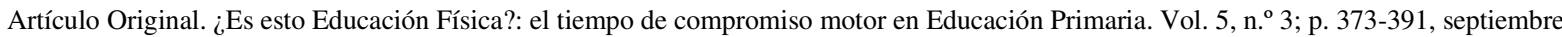
2019. A Coruña. España ISSN 2386-8333.
\end{abstract}

\title{
Introducción
}

Generalmente, en la etapa de EP las sesiones suelen ser de 45 minutos, de modo que el ritmo de trabajo en cuanto a tiempo se refiere, es vertiginoso desde el inicio de la jornada laboral. De acuerdo con lo anterior, se entiende que la gestión del tiempo es una de las preocupaciones más visibles de los docentes (Davini, 2015). Dentro del área de EF sucede lo mismo y desde el punto de vista de la salud, hay que gestionarlo de la mejor forma posible, ya que tal y como afirma Fernández-Revelles (2008) en la actualidad, debido a un estilo de vida más sedentario y a peores hábitos alimenticios, surgen problemas como la obesidad. De hecho, según el estudio de Sánchez-Cruz, Jiménez-Monleón, Fernández-Quesada y Sánchez (2013) sobre la obesidad infantil y juvenil en España, un 40\% tenía sobrepeso u obesidad.

Tanto la obesidad como el sobrepeso infantil suponen ya un trastorno nutricional frecuente a nivel mundial, principalmente en los países desarrollados. Entre ellos España, donde, según un estudio realizado por la International Sport and Culture Association (ISCA, 2015) un $7,4 \%$ de las muertes son causadas por la inactividad, que ocasiona un coste de 990 millones de euros al año en salud. Su impacto puede ocasionar, según la Organización Mundial de la Salud (OMS, 2010), una variedad de complicaciones de salud graves y un mayor riesgo de padecer enfermedades como la diabetes y las cardiopatías prematuramente. Estas consecuencias, negativas para el desarrollo y la calidad de vida de las personas que las padecen, afectan ya no solo al ámbito físico, sino también a la adaptación social y el desarrollo psicológico del sujeto (Santos, 2005).

De este modo, se entiende que tanto la administración educativa en colaboración con la sanitaria de forma general, como los profesionales de la EF en particular, debemos dar respuesta a este problema. En el contexto escolar esta situación se traduce, de acuerdo con Yanci, Vinuesa, Rodríguez y Yanci (2016) y el informe Eurydice de la Comisión Europea (2013), en que un $80 \%$ del alumnado de esta etapa educativa únicamente participa en actividades físicas en el centro escolar. Por lo que es muy importante la motivación que el

Para citar este artículo utilice la siguiente referencia: López-Tavares, A.; Moya-Mata, I. (2019). ¿Es esto Educación Física?: el tiempo de compromiso motor en Educación Primaria. Sportis Sci J, 5 (3), 373-391. DOI: https://doi.org/10.17979/sportis.2019.5.3.5238 


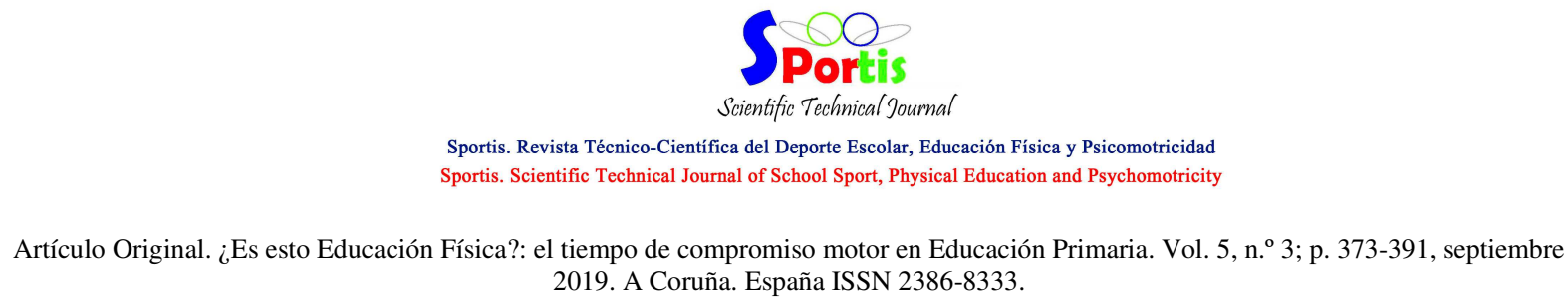

profesorado consiga provocar en su alumnado hacia la práctica de actividad física (MartínezBaena, Mayorga-Vega y Viciana, 2016).

Por ello, conforme a lo expuesto en la introducción del área de EF, recogida en el Real Decreto 126/2014, de 28 de febrero, por el que se establece el currículo básico de la Educación Primaria (BOE, 2014), se debe tener en cuenta que esta ha de ocupar un espacio importante en la jornada lectiva si se pretende ayudar a prevenir y paliar los factores de riesgo que influyen en la aparición de las enfermedades mencionadas anteriormente.

Lo anteriormente descrito acrecienta todavía más el valor que tiene el tiempo en esta área, no solo por su componente motriz, sino por su capacidad para educar en hábitos saludables de higiene y alimentación a los jóvenes, ya que, adquirir estas pautas durante la infancia y la adolescencia tiene una gran influencia sobre la salud en la vida adulta (Cantero, Mayor Toja y González, 2019).

Centrándonos en el marco teórico previo al estudio es necesario destacar algunos conceptos para poder emplear el vocabulario adecuado en cada situación específica. Así, varios autores han establecido diferentes divisiones de la sesión de EF según la gestión del tiempo. Olmedo (2000), partiendo de las ideas de Pierón, realizó la división del tiempo de clase siguiendo una progresión decreciente (efecto embudo) en cinco categorías, de las que se seleccionan las cuatro siguientes: “Tiempo de Programa” (TP), “Tiempo Útil” (TU), “Tiempo Disponible para la Práctica” (TD) y “Tiempo de Compromiso Motor” (TCM) (Figura no 1). Descartando el “Tiempo Empleado en la Tarea”, ya que no es objeto de este estudio. 


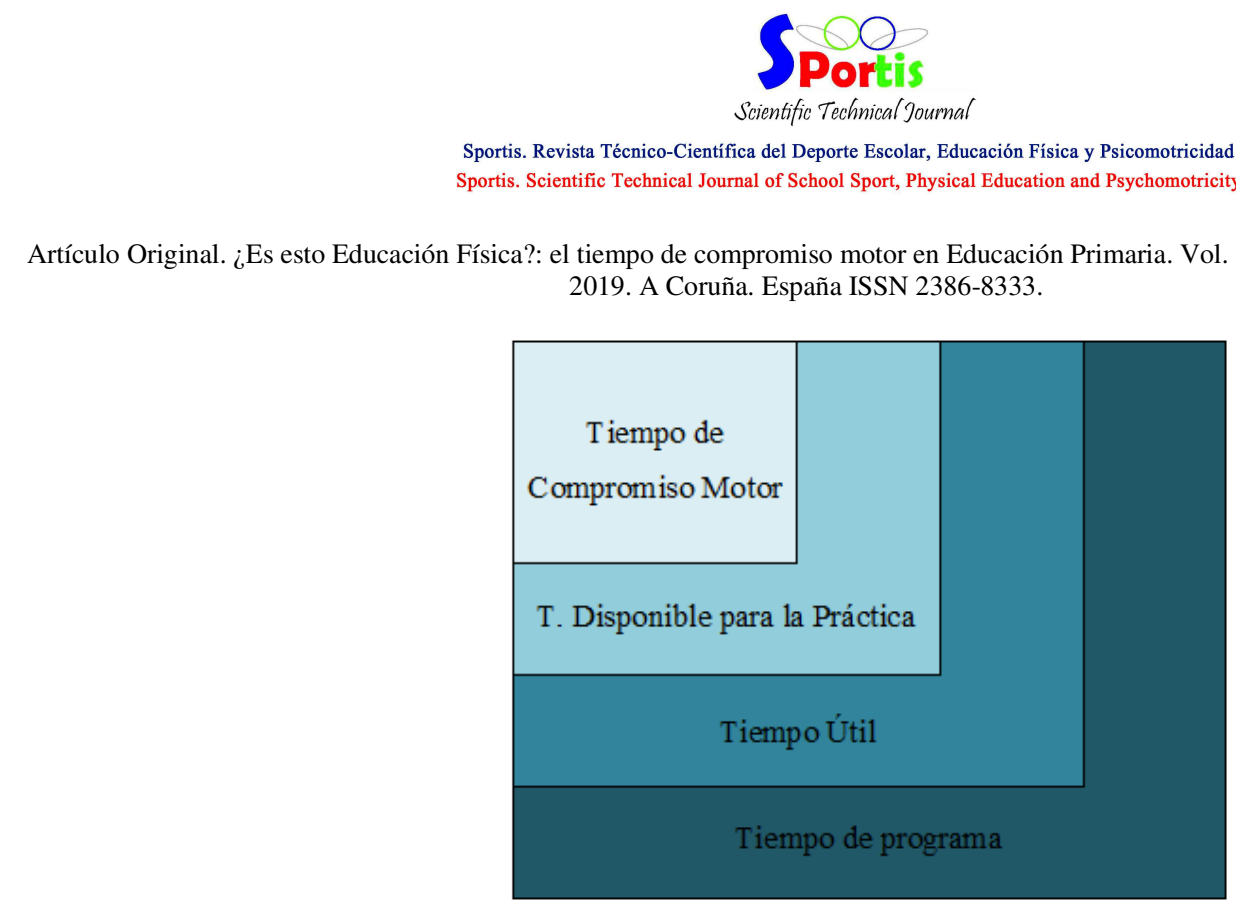

Figura 1. Resumen de la reducción de los tiempos en la sesión de EF basada en Olmedo (2000)

- Tiempo de Programa: tiempo asignado en el horario escolar conforme a lo pautado en la vigente ley educativa. Representa la totalidad del tiempo de la clase.

- Tiempo Útil: tiempo resultante de restar el tiempo de desplazamiento hasta el patio o el gimnasio y la preparación del alumnado. En otras palabras, es el periodo disponible para la práctica desde el inicio de la sesión hasta que finaliza.

- Tiempo Disponible para la Práctica: intervalo temporal del que se dispone una vez que se han dado las explicaciones oportunas y organizado al alumnado y el material.

- Tiempo de Compromiso Motor: tiempo en el cual los sujetos se encuentran en movimiento. El porcentaje recomendable estaría entre el 50-60\% del TD. Según García y Ruiz (2017) es el tiempo que los alumnos pasan haciendo actividades que requieran esfuerzo físico durante la sesión.

En resumen, integrando estos conceptos, se podría definir TCM como el espacio de tiempo derivado del TU tras restarle: el Tiempo de Atención (TA), entendido como el tiempo planificado por el docente en el que va a requerir la atención del alumnado, el Tiempo de Organización (TO), entendido como el tiempo planificado por el docente para organizar al alumnado y el material y el Tiempo Imprevisto (TI) si lo hubiese, entendido como el tiempo

Para citar este artículo utilice la siguiente referencia: López-Tavares, A.; Moya-Mata, I. (2019). ¿Es esto Educación Física?: el tiempo de compromiso motor en Educación Primaria. Sportis Sci J, 5 (3), 373-391. 
Sportis. Revista Técnico-Científica del Deporte Escolar, Educación Física y Psicomotricidad

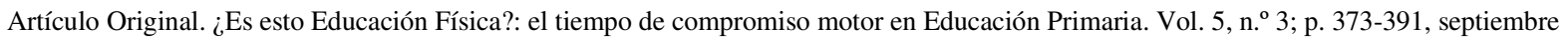
2019. A Coruña. España ISSN 2386-8333.

restante, no organizado ni planificado, durante el cual el alumnado realiza actividad física (existe movimiento) sea o no la ordenada o prevista por el maestro.

Una vez definido el concepto TCM, constatamos como en la sesión de EF se identifican varios factores que lo condicionan, entre ellos destacamos: las agrupaciones, el estilo de enseñanza empleado por el docente, las actividades planteadas, el horario de clase (cuando se da la sesión), la disponibilidad y estado de las instalaciones, el clima motivacional, la edad y la habilidad, entre otros (Yanci et al., 2016).
\end{abstract}

A continuación, presentamos los estudios precedentes a nuestra investigación, los cuales nos han servido para realizar el diseño de este estudio, de manera resumida:

Fernández-Revelles (2008) midió la competencia docente tiempo con un software desarrollado por él mismo. Escogió como muestra un grupo de alumnos (no se especifica edad) durante una sesión de EF y la filmó para que posteriormente fuese revisada por observadores profesionales. Los resultados arrojados mostraron que, en un TP de 60 minutos, quedaban 50 de TU que se reducían a menos de 15 minutos (14:23) de TCM, lo que supone un 23,97\% del TCM respecto al TP y un 28,77\% en relación con el TU.

Yanci et al. (2016) en un estudio de mayor escala, en el que participaron 264 alumnos y alumnas de $1^{\circ}, 2^{\circ}$ y $3^{\circ}$ de primaria, registró un total de 176 sesiones de 50 minutos de TP. Para ello, un único observador entrenado utilizó como herramienta observacional el Tiempo Invertido en la Práctica de Educación Física (TiPEF) compuesta por cuatro criterios (curso, hora de práctica, tipo de sesión y tiempo de compromiso motor) y 19 categorías. Obteniendo que la duración media del TCM para todas las sesiones analizadas fue de 21,4 $\pm 3,8$ min./sesión.

El alumnado de los tres primeros cursos de EP dispuso de un tiempo de práctica de actividad física de un 42,8\% con respecto al TP, lo que supone que un 57,2\% del tiempo se destinó a otras tareas que no implicaron práctica motriz. En total, los sujetos del estudio no realizaron más de 45 minutos semanales de práctica motriz en las clases de EF. El TCM fue

Para citar este artículo utilice la siguiente referencia: López-Tavares, A.; Moya-Mata, I. (2019). ¿Es esto Educación Física?: el tiempo de compromiso motor en Educación Primaria. Sportis Sci J, 5 (3), 373-391. DOI: https://doi.org/10.17979/sportis.2019.5.3.5238 


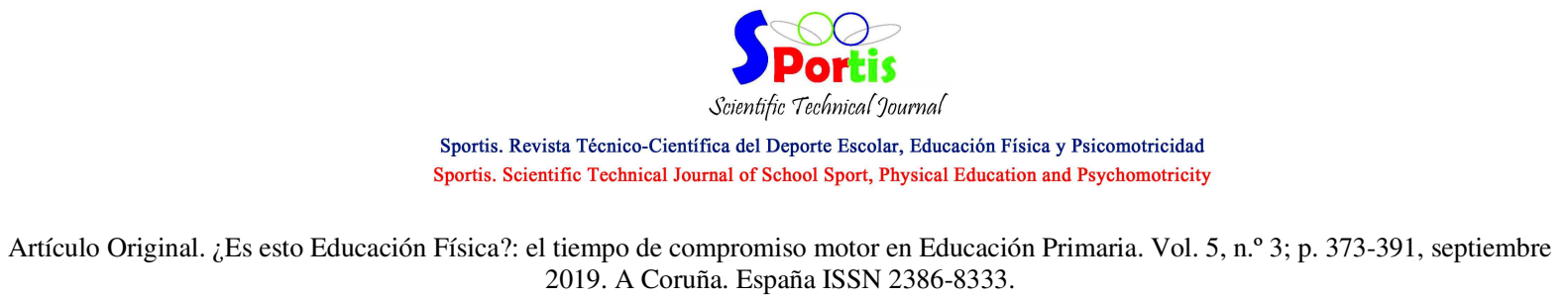

significativamente distinto en función de la edad. Los alumnos de primer curso dispusieron de mayor cantidad de tiempo de práctica por sesión en comparación con los de tercer curso, que fueron los que menos tiempo de práctica realizaron.

En su estudio, Martínez-Gómez, Sampedro-De la Granja y Veiga Núñez (2007) tienen como objetivo descubrir qué metodología (instrucción directa e indagación o búsqueda) es más eficaz para conseguir mayor compromiso motor. Para su estudio escogieron una muestra de 27 alumnos de $6^{\circ}$ de primaria. Para medir el TCM, dos doctores en Ciencias de la Actividad Física y el Deporte con experiencia en la investigación observacional, registraron in situ los tiempos que se producía en la sesión y la duración de cada uno de ellos en dos sesiones de 55 minutos, cada una con un estilo de enseñanza. De este modo, se muestra el mayor TCM en la sesión que utiliza la indagación o búsqueda como técnica de enseñanza llegando al 47,12\% del tiempo de programa, frente a la sesión basada en la instrucción directa $(35,42 \%)$.

Por su parte, el estudio de Gracia y Ruiz (2017) tiene como principal objetivo conocer la cantidad de TCM en una muestra de 120 alumnos de $1^{\circ}, 3^{\circ}$ y $4^{\circ}$ de ESO y $1^{\circ}$ de Bachillerato con respecto al total del tiempo programado. Como instrumentos para la observación y obtención de datos utilizaron: un cronómetro y unas hojas de registro para anotar los tiempos que se sucedían durante la sesión según su tipología. La toma de datos se realizó mediante observación directa externa, teniendo en cuenta al grupo como conjunto y no al sujeto de forma individual. En total, se observaron 10 sesiones de grupos de nivel académico diferente y de diferentes contenidos: cuatro sesiones de waterpolo (dos de $4^{\circ}$ ESO y dos de $1^{\circ}$ Bachillerato), cuatro sesiones de atletismo (dos de $1^{\circ}$ ESO y dos de $3^{\circ}$ ESO) y dos sesiones de voleibol ( $1^{\circ}$ de Bachillerato) con un TP de 50 minutos. Así, el tiempo de TCM medio obtenido en todas las clases observadas es del 48,22\% (24,11 min./sesión).

Por lo tanto, una vez analizados los estudios precedentes, el objetivo de la presente investigación es analizar el TCM en función de la sesión y el curso escolar en el alumnado de primer y quinto curso de EP.

Para citar este artículo utilice la siguiente referencia: López-Tavares, A.; Moya-Mata, I. (2019). ¿Es esto Educación Física?: el tiempo de compromiso motor en Educación Primaria. Sportis Sci J, 5 (3), 373-391. DOI: https://doi.org/10.17979/sportis.2019.5.3.5238 


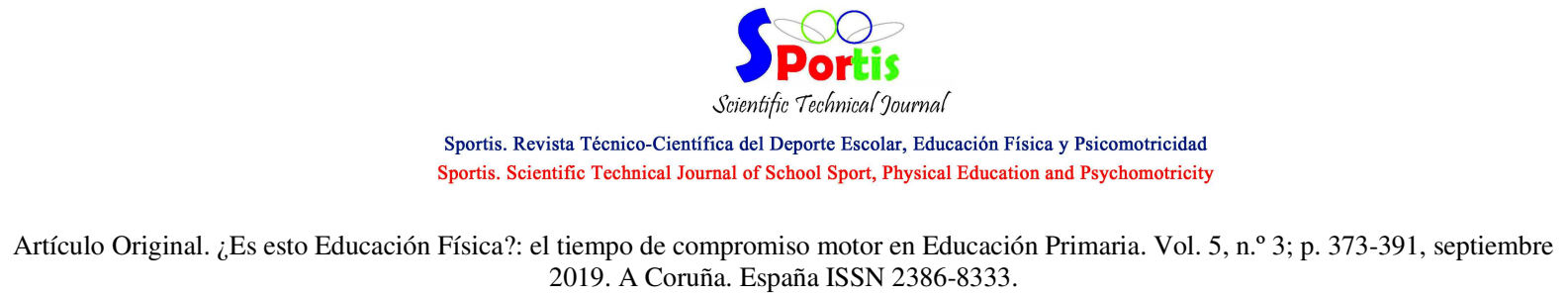

\section{Material y método}

\section{Diseño del estudio}

El diseño empleado en la investigación fue ex post-facto (Ato, López y Benavente, 2013), al no existir intervención por parte del investigador y siendo el objetivo último la descripción de aquello que está ocurriendo en las clases de EF, teniendo por tanto un carácter descriptivo. La metodología planteada es cuantitativa (Thomas y Nelson, 2007) y comparativa entre los cursos seleccionados.

\section{Muestra}

En el estudio participaron 38 participantes, 18 del primer curso y 20 del quinto curso, de seis y 10 años respectivamente, de EP, durante el curso escolar 2017-18 de un colegio público de la Comunidad Valenciana. El motivo de la elección de la muestra se debe a la diferencia de edad que existe entre el alumnado de primero y de quinto de EP, y los estudios precedentes sobre el TCM en Primaria, ya que sí que existen estudios con alumnado de $1^{\circ}$ curso, pero no de $5^{\circ}$ de EP. Los dos grupos realizaban dos sesiones de EF semanal con una duración de 45 minutos por sesión, dirigidas por el docente especialista del centro.

\section{Instrumento}

Para el registro de los datos se diseñó una herramienta observacional basada en el sistema de registro de las variables temporales de Ramírez, Lozano, San Matías, Zabala y Viciana (2003), y la nomenclatura de Olmedo (2000), como se muestra en la Figura 2. 
Artículo Original. ¿Es esto Educación Física?: el tiempo de compromiso motor en Educación Primaria. Vol. 5, n. ${ }^{\circ}$ 3; p. 373-391, septiembre 2019. A Coruña. España ISSN 2386-8333.

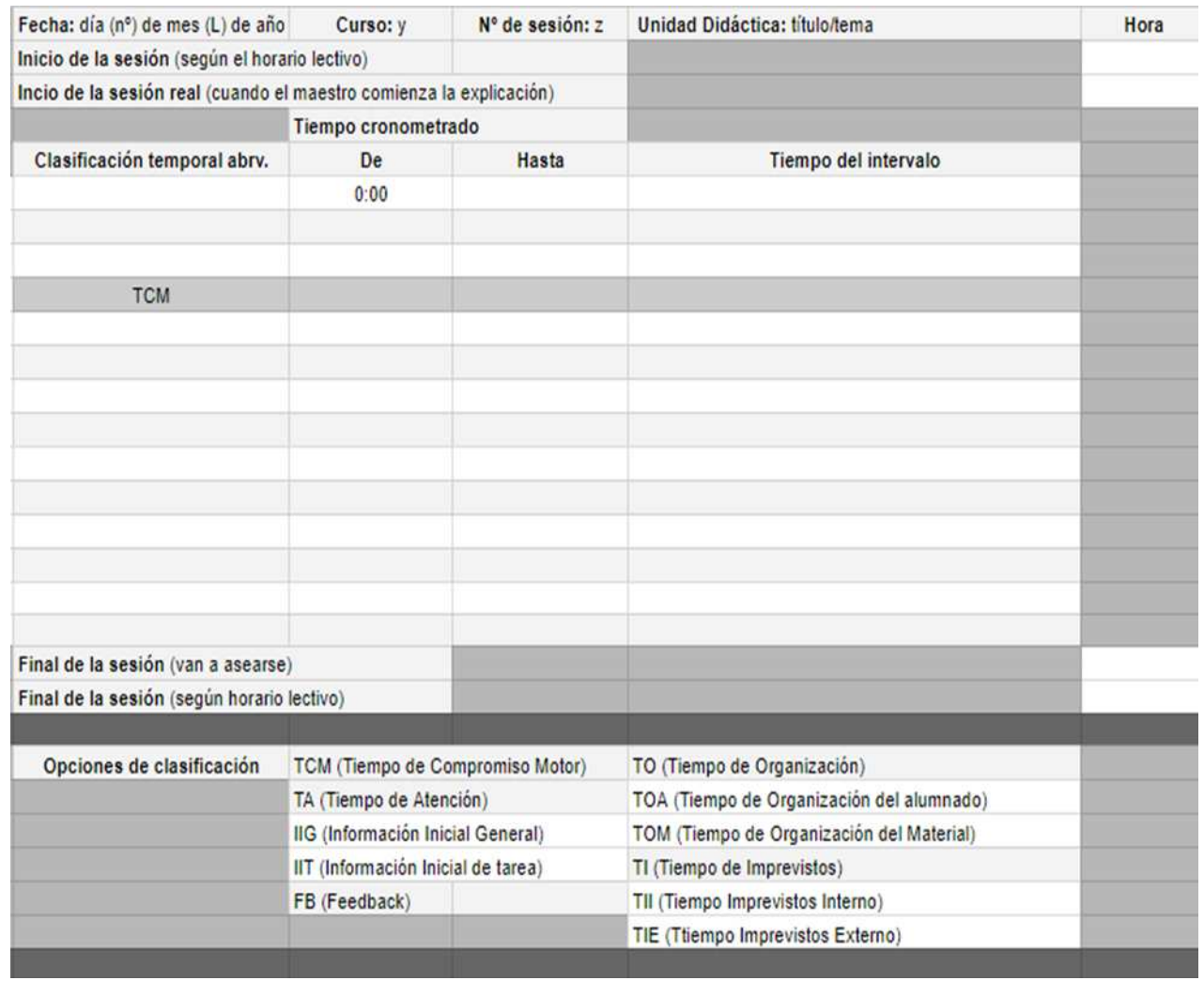

Figura 2. Modelo de hoja de registro basado en Ramírez et al. (2003) y Olmedo (2000).

Los ítems que aparecen en la parte inferior se fueron contabilizando y anotando en los apartados correspondientes de la hoja de registro, junto a los tiempos de cada intervalo (medidos en el formato mm:ss), mediante el cronómetro instalado en un dispositivo móvil. Asimismo, se tomaron referencias sobre la hora de inicio de la sesión y el final de ésta con el objetivo de obtener el TU de la sesión.

\section{Procedimiento}

Se grabaron un total de nueve sesiones de EF, supervisadas y dirigidas por uno de los docentes especialistas del centro educativo. De éstas, cuatro sesiones correspondieron a la Unidad Didáctica de equilibrio del grupo de $1^{\circ}$, y cinco sesiones a la Unidad Didáctica de atletismo de $5^{\circ}$. 

2019. A Coruña. España ISSN 2386-8333.

Las sesiones fueron grabadas desde un punto externo a la zona de actividad física para mostrar una vista periférica y que no supusiese un elemento que condicionara la práctica de los alumnos. En las esquinas de las pistas, en los porches y en las esquinas del gimnasio se situaron los puntos para tomar imágenes, intentando en todo momento restar importancia a la presencia del observador en el campo de la investigación.
\end{abstract}

En lo referente al registro de datos, antes de comenzar, se dejaron claras las premisas, los criterios y las categorías, sin dejar margen a la ambigüedad. Así pues, ante la coincidencia de dos ítems de forma simultánea se aplicaría el que más cantidad de sujetos concentre o se tomaran ambos si no se trata de TCM. Por ejemplo, la organización del alumnado y el material al unísono. Actividades como los relevos, en las que se forman filas y la actividad motriz no es continua, serán consideradas como TCM. Del mismo modo, cuando se produce un tiempo de feedback y de TCM sin que el primero suponga la interrupción del segundo, se considerará solamente este último. La rutina establecida para la grabación de las sesiones constaba de dos partes:

- En primer lugar, tras la preparación del material (montaje del trípode y comprobación de la funcionalidad de la cámara), minutos antes del inicio de la sesión, se colocaba el observador en uno de los puntos establecidos para la grabación con el material necesario para iniciarla. La grabación no comenzaba hasta que se iniciaba la hora prevista según el horario escolar para la sesión (independientemente de que el grupo o el docente se encontraran o no en el patio).

- La segunda parte comenzaba con el inicio de la sesión y, por ende, de la grabación. Ésta consistía en filmar la sesión al completo hasta el momento en el que el maestro la diese por concluida, ordenando la tarea de asearse.

Aunque la mayor parte del TP se trabajó en la pista, el espacio varió de una sesión a otra y también dentro de una misma sesión. En estos casos, se seguía el desplazamiento de los 

2019. A Coruña. España ISSN 2386-8333.

componentes de la clase hasta colocarse el observador en otro de los puntos nombrados anteriormente.
\end{abstract}

Para llevar a cabo nuestra investigación se solicitó permiso al docente de EF y a la dirección del Centro, que dieron el visto bueno tras haber sido informados del procedimiento. Para actuar dentro de la legalidad, cumpliendo con el respeto a la intimidad de las personas y de los colectivos, en conformidad con las normas de protección de datos, se contó con la autorización de los padres, madres o tutores legales a través del centro escolar para que se pudieran tomar imágenes y vídeos de los menores para este estudio, firmando el preceptivo consentimiento informado. El estudio fue aprobado por el Consejo Escolar del centro educativo, cumpliendo con la Declaración de Helsinki (2013).

El análisis descriptivo del cual se extrajo el porcentaje de frecuencia del TCM de los dos cursos del estudio se realizó mediante el programa Statistical Package for Social Sciences (SPSS $®$, versión 21.0) para Windows. Licencia del Campus de la Universidad de Valencia. Se realizó un análisis descriptivo univariable y los resultados se presentaron en porcentajes.

\title{
Resultados
}

En $1^{\circ}$ de EP, con un TP de 45 minutos, se obtuvo un TCM medio de 12:50 minutos/sesión en la unidad didáctica de equilibrios (28,53\%); siendo la primera sesión donde se alcanzó el máximo TCM, con 14:07 minutos/sesión (31,37\% del TP), y la tercera sesión donde se alcanzó el mínimo TCM con 10:38 minutos/sesión (23,63\% del TP).

En cuanto al TU se observan valores estables (3 minutos de diferencia máximo entre sesiones), siendo el TU medio de 32:45 minutos, lo que supone una pérdida de un 27,2\% con relación al TP (Tabla $\left.n^{\circ} 1\right)$.

Asimismo, se extrajeron los porcentajes por sesión y el porcentaje medio de TCM respecto al TU.

Para citar este artículo utilice la siguiente referencia: López-Tavares, A.; Moya-Mata, I. (2019). ¿Es esto Educación Física?: el tiempo de compromiso motor en Educación Primaria. Sportis Sci J, 5 (3), 373-391. DOI: https://doi.org/10.17979/sportis.2019.5.3.5238 
Tabla $n^{\circ} 1$. Frecuencias de los datos recogidos de las sesiones de $1^{\circ}$ curso

\begin{tabular}{|c|c|c|c|c|c|}
\hline $\begin{array}{l}\text { Cur } \\
\text { so }\end{array}$ & $\begin{array}{c}\mathbf{N}^{\mathbf{o}} \text { de } \\
\text { sesion } \\
\text { es }\end{array}$ & $\begin{array}{c}\text { TU } \\
\text { (minut } \\
\text { os) }\end{array}$ & $\begin{array}{c}\text { TCM } \\
(\mathbf{m m}: \\
\text { ss) }\end{array}$ & $\begin{array}{c}\text { TCM/ } \\
\text { TP } \\
(\%)\end{array}$ & $\begin{array}{c}\text { TCM/ } \\
\text { TU } \\
(\%)\end{array}$ \\
\hline \multirow{4}{*}{$1^{\circ}$} & 1 & 32 & $14: 07$ & 31,37 & 44,11 \\
\hline & 2 & 34 & $12: 43$ & 28,26 & 37,40 \\
\hline & 3 & 31 & $10: 38$ & 23,63 & 34,30 \\
\hline & 4 & 34 & $13: 54$ & 30,08 & 43,82 \\
\hline \multicolumn{2}{|c|}{ Media Resultados } & $32: 45$ & $12: 50$ & 28,53 & 39,19 \\
\hline
\end{tabular}

$\mathrm{TP}=$ Tiempo de Programa, $\mathrm{TU}=$ Tiempo Útil, $\mathrm{TCM}=$ tiempo de compromiso motor, $\min =$ minutos, $\mathrm{mm}: \mathrm{ss}=$ minutos y segundos.

En $5^{\circ}$ de EP, con un TP también de 45 minutos, los resultados extraídos muestran que el TCM medio en la Unidad Didáctica de atletismo fue del 27,05\%, es decir, de 12:10 min./sesión. Siendo la cuarta sesión donde se alcanzó el máximo TCM, con 16:24 minutos/sesión (36,44\% del TP), y la primera sesión donde se alcanzó el mínimo TCM con 8:29 minutos/sesión (18,8\% del TP).

El TU medio de este curso fue de 35:12 minutos, lo que refleja que, de media, hay aproximadamente 10 minutos de pérdida por clase, descontando los desplazamientos hasta el comienzo de la sesión y el tiempo para la higiene personal. De igual modo que para el primer curso, se calcularon los porcentajes por sesión y el porcentaje medio de TCM respecto al TU $\left(\right.$ Tabla $\left.n^{0} 2\right)$.

Tabla $n^{\circ} 2$. Frecuencias de los datos recogidos de las sesiones de $5^{\circ}$ curso

\begin{tabular}{|c|c|c|c|c|c|}
\hline $\begin{array}{l}\text { Cur } \\
\text { so }\end{array}$ & $\begin{array}{c}N^{0} \text { de } \\
\text { sesion } \\
\text { es }\end{array}$ & $\begin{array}{c}\text { TU } \\
\text { (minut } \\
\text { os) }\end{array}$ & $\begin{array}{c}\text { TCM } \\
\text { (mm: } \\
\text { ss) }\end{array}$ & $\begin{array}{c}\text { TCM/ } \\
\text { TP } \\
(\%)\end{array}$ & $\begin{array}{c}\text { TCM/ } \\
\text { TU } \\
(\%)\end{array}$ \\
\hline \multirow{5}{*}{$5^{\circ}$} & 1 & 35 & $08: 29$ & 18,80 & 24,24 \\
\hline & 2 & 32 & $10: 29$ & 23,30 & 30,83 \\
\hline & 3 & 37 & $12: 19$ & 27,37 & 38,49 \\
\hline & 4 & 38 & $16: 24$ & 36,44 & 44,32 \\
\hline & 5 & 34 & $13: 12$ & 29,33 & 34,74 \\
\hline \multicolumn{2}{|c|}{ Media Resultados } & $35: 12$ & $12: 10$ & 27,05 & 34,52 \\
\hline
\end{tabular}

$\mathrm{TP}=$ Tiempo de Programa, $\mathrm{TU}=$ Tiempo Útil, $\mathrm{TCM}=$ tiempo de compromiso motor, $\min =$ minutos, $\mathrm{mm}: \mathrm{ss}=$ minutos y segundos.

Para citar este artículo utilice la siguiente referencia: López-Tavares, A.; Moya-Mata, I. (2019). ¿Es esto Educación Física?: el tiempo de compromiso motor en Educación Primaria. Sportis Sci J, 5 (3), 373-391. 


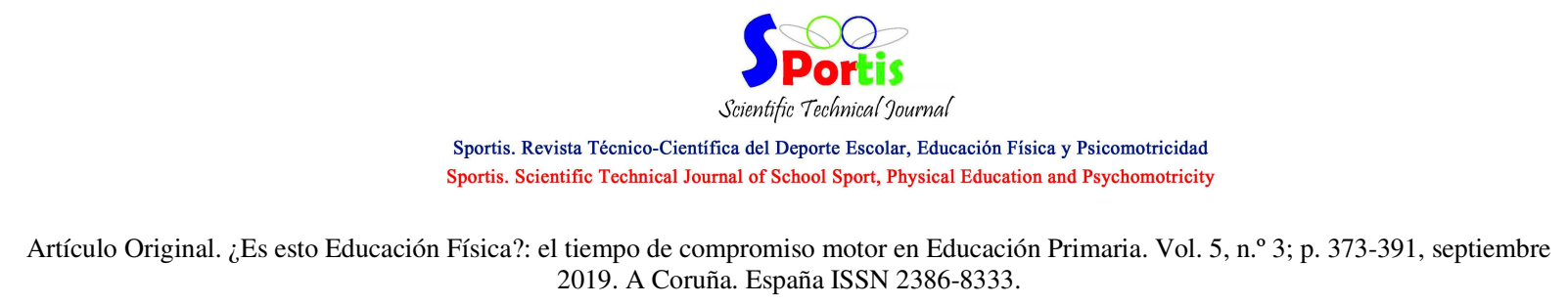

\section{Discusión}

El principal objetivo de nuestra investigación fue averiguar la relación entre el TCM de la sesión y la edad del alumnado de primer y quinto curso de EP.

Por un lado, el porcentaje medio de TCM de la clase de $1^{\circ}$ fue inferior al $30 \%$. Valores que, en comparación con los mostrados en investigaciones similares como la de FernándezRevelles (2008) se sitúan ligeramente por encima. Sin embargo, si lo comparamos con el estudio de Yanci et al. (2016) son significativamente inferiores, al encontrar un TCM del $42,8 \%$.

Por otro lado, los resultados obtenidos en $5^{\circ}$ curso, también nos muestran un TCM menor con respecto a otras investigaciones, como es el estudio de Martínez-Gómez et al. (2007) en $6^{\circ}$ de EP, donde resulta ser del 35,42\% y 47,12\%, según el método de enseñanza empleado; o el estudio de Gracia y Ruiz (2017), en la etapa de Educación Secundaria Obligatoria, donde el TCM fue del 48,22\%.

Aunque el TCM de los alumnos de $1^{\circ}$ es mayor con respecto a los alumnos de $5^{\circ}$ de EP, siendo la diferencia entre el máximo y el mínimo TCM en $1^{\circ}$ curso de menos de cuatro minutos, mientras que, en $5^{\circ}$ curso, la diferencia es de casi ocho minutos; en estudios como el de Gracia y Ruiz (2017) no se encuentran diferencias entre diferentes niveles educativos. Y es que la observación directa de las sesiones, así como el visionado de los vídeos nos indican que una buena motivación (encontrada en el alumnado de menor edad) y una buena gestión de la clase por parte del docente con respecto a las agrupaciones y la metodología empleada en cada situación, es fundamental para optimizar el TCM del alumnado.

Otra de las diferencias entre ambos cursos es que $5^{\circ}$ goza de mayor TU (35:12 minutos). A pesar de ello, tiene menos TCM/TP $(27,05 \%)$ que $1^{\circ}(28,53 \%)$; la diferencia no es muy amplia pero sí es considerable. Esta aumenta si se tienen en consideración los valores porcentuales que relacionan el TCM con el TU (34,52\% y 39,19\% respectivamente) debido a que, como se señala anteriormente, el TU es mayor en el caso de $5^{\circ}$ curso. En definitiva, se

Para citar este artículo utilice la siguiente referencia: López-Tavares, A.; Moya-Mata, I. (2019). ¿Es esto Educación Física?: el tiempo de compromiso motor en Educación Primaria. Sportis Sci J, 5 (3), 373-391.

DOI: https://doi.org/10.17979/sportis.2019.5.3.5238 

2019. A Coruña. España ISSN 2386-8333.

cumple, como ocurre en el estudio de Yanci et al. (2016), que los alumnos de menor edad tienen más TCM.
\end{abstract}

Pero estos datos no son suficientes para paliar el sedentarismo, tal y como expone el Real Decreto 126/2014 (BOE, 2014) en el área de EF, en donde además se establece que la EF en EP debe tener una presencia importante en la jornada escolar.

Es necesario remarcar la importancia del tiempo de actividad física en EF, ya que, de acuerdo con Carretero et al. (2017), se trata de la única área curricular en la que existe movimiento por parte de un alumnado cada vez más sedentario. Es decir, la EF educa por y para una vida saludable persiguiendo el desarrollo integral de la persona (físico, mental y social) y promoviendo valores sociales básicos para la convivencia (cooperación, juego limpio, etc.).

\title{
Conclusiones
}

El TCM realizado por el alumnado de primero y quinto curso de EP no es suficiente para obtener beneficios para la salud y el bienestar físico, mental y social; ya que como estima el informe Eurydice de la Comisión Europea (2013), hasta un 80\% del alumnado en edad escolar únicamente participan en actividades físicas en la escuela.

El tiempo lectivo sobre el área de EF que marca la normativa legal española es insuficiente para abordar los contenidos y cumplir los objetivos que se plantean. Hecho que invita a la reflexión sobre el número semanal de sesiones y el tiempo de cada una de éstas dentro del horario escolar.

Por todo ello, tanto la administración educativa como los docentes y profesionales del ámbito de la EF han de cooperar con el fin de elaborar acciones específicas a pequeña y gran escala, encaminadas a aumentar el TCM en estas edades.

Como se ha podido observar, los datos obtenidos son mejorables en cuanto a valores de TCM se refiere, en comparación con algunos de los estudios previos mencionados en el Para citar este artículo utilice la siguiente referencia: López-Tavares, A.; Moya-Mata, I. (2019). ¿Es esto Educación Física?: el tiempo de compromiso motor en Educación Primaria. Sportis Sci J, 5 (3), 373-391. 


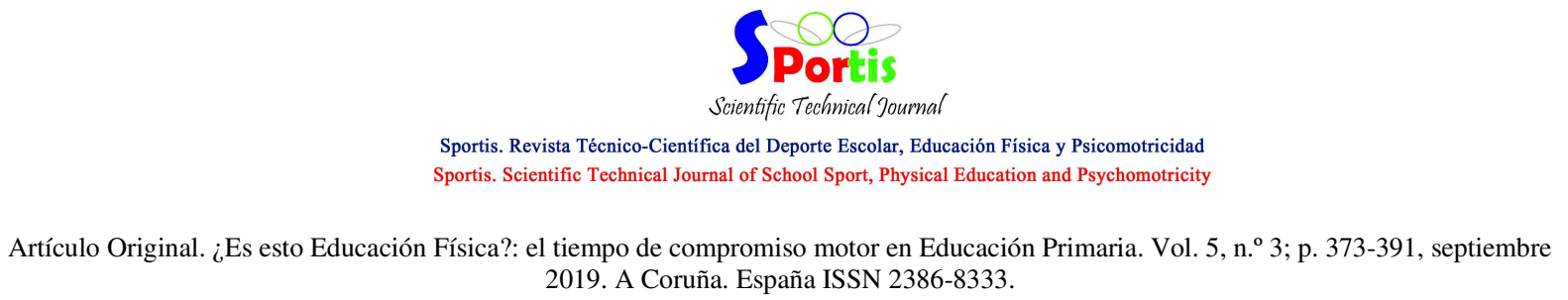

apartado de la discusión. Para ello, de acuerdo con investigadores como Olmedo (2000), Sierra (2003) y Abad y Cañada (2017), además de aportaciones propias fruto de la observación, se recopilan, de forma resumida, algunas estrategias para aumentar el tiempo de actividad motriz del alumnado clasificadas en las siguientes categorías:

\section{$\underline{\text { Motivación }}$}

- Averiguar y tener en cuenta los gustos (juegos preferidos) del grupo clase.

- Desplazarse por el espacio animando al alumnado frecuentemente, con el objetivo de potenciar su participación de forma activa en las actividades.

- Utilizar diversidad de materiales atractivos y sencillos.

- Diseñar, seleccionar y plantear actividades con metas que los alumnos sean capaces de alcanzar en relación con sus habilidades.

- Creación de un sistema gamificado que refuerce la participación para lograr una mayor implicación de la clase. Para ello se podría recurrir a la utilización de una moneda saludable ("healthies") en función de la implicación motriz del grupo-clase. Los "healthies" obtenidos se podrán canjear por recompensas que sigan promoviendo actividades físicas.

\section{Organización}

- Organizar actividades de manera que la ejecución de los alumnos sea simultánea y/o alternativa, evitando que el alumnado se mantenga gran parte del tiempo estático, propiciando así, que la mayoría de la clase esté en movimiento al mismo tiempo.

- Establecer grupos homogéneos de nivel dentro de la clase para favorecer la participación de todos los integrantes del grupo-clase.

\footnotetext{
Para citar este artículo utilice la siguiente referencia: López-Tavares, A.; Moya-Mata, I. (2019). ¿Es esto Educación Física?: el tiempo de compromiso motor en Educación Primaria. Sportis Sci J, 5 (3), 373-391. DOI: https://doi.org/10.17979/sportis.2019.5.3.5238
} 


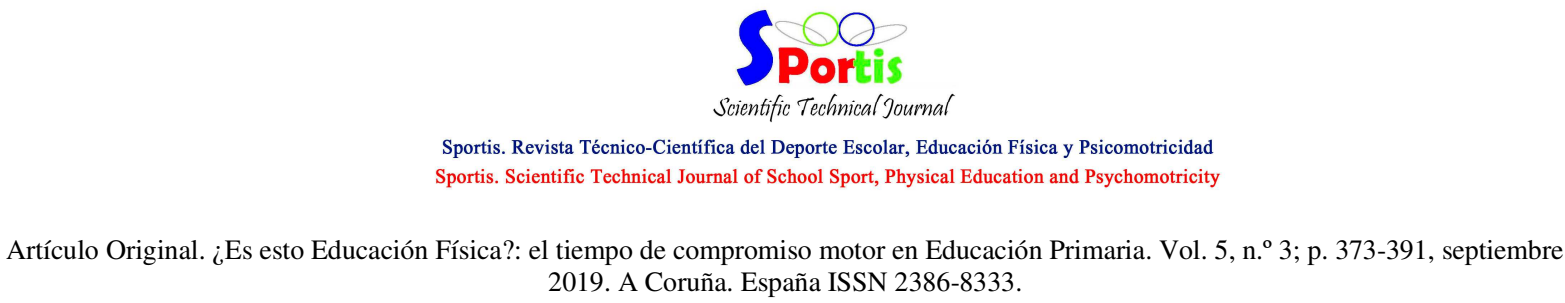

- Utilizar variantes de un mismo ejercicio aprovechando el material para reducir el Tiempo de Organización del Alumnado y el Tiempo Organización del Material.

- Preparar y disponer del material que se va a necesitar con antelación. De forma que, la cantidad, según el número de alumnos, sea suficiente para que la sesión resulte lo más participativa posible.

- Tener en cuenta las dimensiones del espacio y el número de participantes en relación con los objetivos del ejercicio.

- Pasar lista durante el trascurso de las actividades.

- Establecer códigos de comunicación (señales visuales y/o acústicas sencillas) como: levantar la mano para pedir silencio; utilizar palabras como "reunión", para que toda la clase se acerque a un punto de encuentro para recibir las explicaciones; si el silbato suena dos veces, el juego ha terminado, con el objetivo de marcar los tiempos de transición.

- Asignación de roles predeterminados a los alumnos, como por ejemplo el de "ayudantes del profesor" para fomentar la participación del alumnado en la organización, colocación y recogida del material.

\section{$\underline{\text { Metodología }}$}

- Uso de estilos de enseñanza abiertos (descubrimiento guiado, resolución de problemas, etc.) que favorecen la actividad física ininterrumpida.

- Explicaciones claras, breves y estructuradas.

- Reducir el margen para la improvisación intentando controlar la mayoría de los elementos que intervienen en la sesión.

\footnotetext{
Para citar este artículo utilice la siguiente referencia: López-Tavares, A.; Moya-Mata, I. (2019). ¿Es esto Educación Física?: el tiempo de compromiso motor en Educación Primaria. Sportis Sci J, 5 (3), 373-391. DOI: https://doi.org/10.17979/sportis.2019.5.3.5238
} 


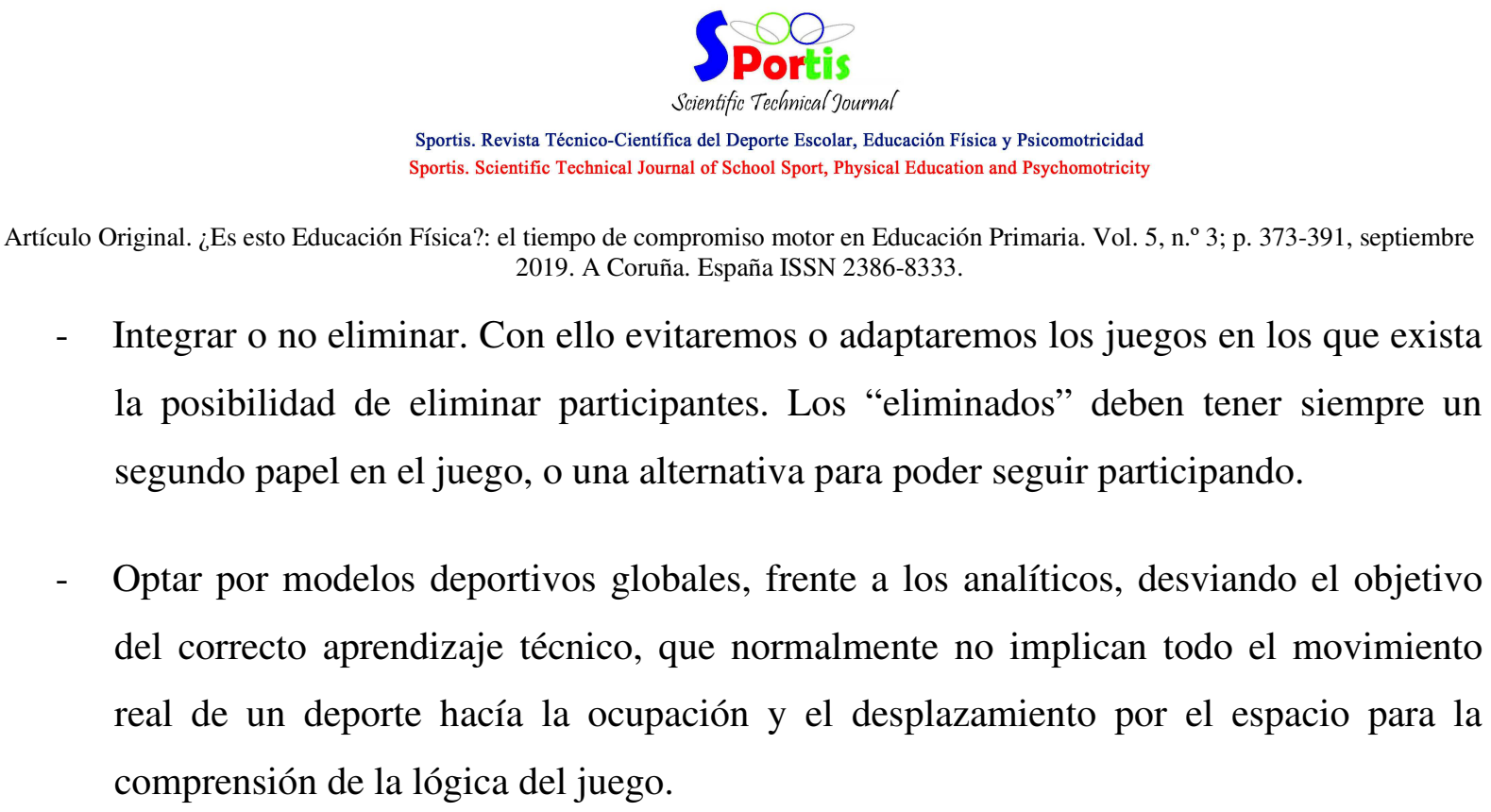

Estas estrategias, accesibles a todo el profesorado, pueden abordarse de forma conjunta para producir efectos todavía más significativos a la hora de incrementar los valores de TCM durante las clases de EF.

\title{
Referencias bibliográficas
}

\author{
Abad, B., y Cañada, D. (2014). Estrategias metodológicas para una Educación Física \\ más activa. Publicaciones Ministerio de Educación Ciencia y Cultura. \\ https://doi.org/10.1174/113564009787531172
}

Ato, M., López, J.J., y Benavente, A. (2013). Un sistema de clasificación de los diseños de investigación en psicología. Anales de Psicología, 29(3), 1038-1059. https://doi.org/10.6018/analesps.29.3.178511

Cantero, P., Mayor, A., Toja, B., y González, M. (2019). Fomento de estilos de vida activos en la escuela: práctica de actividad física, edad y género. Sportis. Scientific Journal of School Sport, Physical Education and Phychomotricity, 5(1), 53-69. https://doi.org/10.17979/sportis.2019.5.1.3680

Carretero, M., Ramos, A.L., Arribas, S., del Castillo, J.M., Hernández, Y., Aparicio, D., ... Díez, C. (2017). ¿Por qué es necesario aumentar las horas de educación física? Revista Española de Educación Física y Deportes, 419, 55-69. https://doi.org/10.5672/apunts.20140983.es.(2014/4).118.04

\footnotetext{
Para citar este artículo utilice la siguiente referencia: López-Tavares, A.; Moya-Mata, I. (2019). ¿Es esto Educación Física?: el tiempo de compromiso motor en Educación Primaria. Sportis Sci J, 5 (3), 373-391. DOI: https://doi.org/10.17979/sportis.2019.5.3.5238
} 


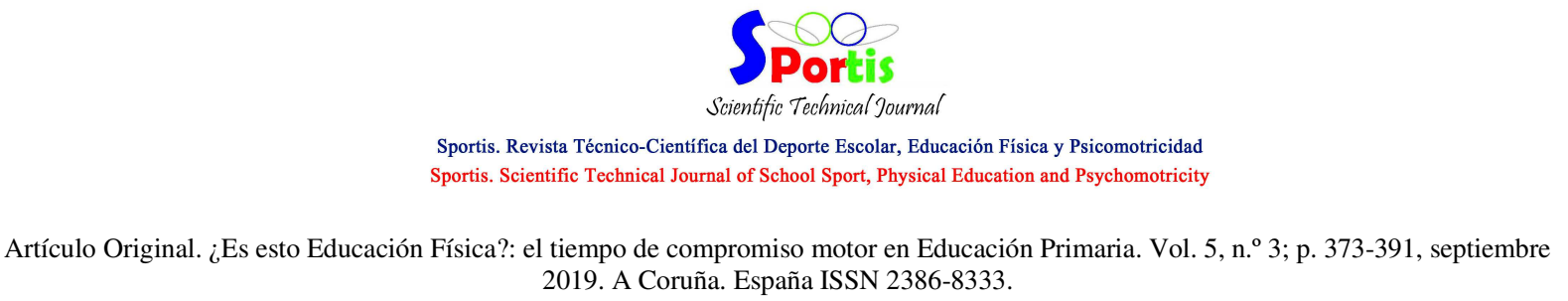

Comisión Europea, EACEA and Eurydice (2013). La educación física y el deporte en los centros escolares de Europa. Informe de Eurydice. Luxemburgo: Oficina de Publicaciones de la Unión Europea. https://doi.org/10.5944/reec.20.2012.7605

Davini, C. (2015). La formación en la práctica docente. Educación, Lenguaje y Sociedad. Buenos Aires: Paidós. 186 págs. ISBN: 978-950-12-0196-3. https://doi.org/10.19137/els-2015-121207

Fernández-Revelles, A.B. (2008). El tiempo en la clase de Educación Física, la competencia docente tiempo. Deporte y Actividad Física para todos, 4, 102-120. https://doi.org/10.24310/riccafd.2018.v7i3.5537

Gracia E., y Ruiz G. (2017). Análisis del tiempo de compromiso motor en Educación Física. EmásF, 8(45), 21-51.

International Sport and Culture Association (ISCA, 2015). The economic cost of physical inactivity in Europe. Recuperado de https://inactivity-timebomb.nowwemove.com/downloadreport/The\%20Economic\%20Costs $\% 20$ of $\% 20$ Physical $\% 20$ Inactivity\%20in\%20Europe\%20(June\%202015).pdf

Martínez-Baena, A., Mayorga-Vega, D., y Viciana, J. (2016). Relación de los niveles de actividad física con el género y el perfil de riesgo cardiovascular en adolescentes granadinos. Implicaciones didácticas para la Educación Física. Profesorado. Revista de currículum y formación del profesorado, 20(1), 265-285.

Martínez-Gómez, D., Sampedro-De la Granja, M.V., y Veiga Núñez, O.L. (2007). La importancia del compromiso motor y el compromiso fisiológico durante las clases de Educación Física. Revista Iberoamericana de Educación, 42(2). https://doi.org/10.30827/profesorado.v23i1.9170

Olmedo, J.A. (2000). Estrategias para aumentar el tiempo de práctica motriz en las clases de Educación Física escolar. Apunts. Educación Física y Deportes, 59, 22-30. https://doi.org/10.5672/apunts.2014-0983.es.(2014/2).116.09

Organización Mundial de la Salud (OMS, 2010). Recomendaciones mundiales sobre actividad física para la salud. https://doi.org/10.1590/s1020-49892001001000014

Ramírez, J., Lozano, L., San Matías, J., Zabala, M., y Viciana, J. (2003). Directrices metodológicas para la observación sistemática del tiempo de clase en la investigación de la educación física. En J. Campos y V. Carratalá (Eds.), Actas del III Congreso de la Asociación Para citar este artículo utilice la siguiente referencia: López-Tavares, A.; Moya-Mata, I. (2019). ¿Es esto Educación Física?: el tiempo de compromiso motor en Educación Primaria. Sportis Sci J, 5 (3), 373-391. DOI: https://doi.org/10.17979/sportis.2019.5.3.5238 
Artículo Original. ¿Es esto Educación Física?: el tiempo de compromiso motor en Educación Primaria. Vol. 5, n. 3; p. 373-391, septiembre 2019. A Coruña. España ISSN 2386-8333.

Española de Ciencias del Deporte. Valencia: Universidad de Valencia. https://doi.org/10.4995/aniav.2017.5165

Real Decreto 126/2014, de 28 de febrero, por el que se establece el currículo básico de la Educación Primaria, 52. B.O.E., 1-58 (1 de marzo de 2014). https://doi.org/10.18002/pec.v0i12.617

Sánchez-Cruz, J.J., Jiménez-Monleón, J.J., Fernández-Quesada, F., y Sánchez, M.J. (2013). Prevalencia de obesidad infantil y juvenil en España en 2012. Revista Española de Cardiología, 66(5), 371-376. https://doi.org/10.1016/j.recesp.2012.10.016

Santos Muñoz, S. (2005). La Educación Física escolar ante el problema de la obesidad y el sobrepeso. Revista Internacional de Medicina y Ciencias de la Actividad Física y el Deporte, 5(19), 179-199. https://doi.org/10.15366/rimcafd2018.72.004

Sierra, A. (2003). Actividad física y salud en primaria: el compromiso fisiológico en la clase de Educación Física. Wanceulen: Sevilla.

Thomas, J.R., y Nelson, J.K. (2007). Métodos de investigación en actividad física. Paidotribo: Barcelona.

Yanci, J., Vinuesa, A., Rodríguez, J., y Yanci, L. (2016). El tiempo de compromiso motor en las sesiones de Educación Física del primer y segundo ciclo de Educación Primaria. Sportis. Scientific Journal of School Sport, Physical Education and Psychomotricity, 2(2), 239-253. https://doi.org/10.17979/sportis.2016.2.2.1447

Para citar este artículo utilice la siguiente referencia: López-Tavares, A.; Moya-Mata, I. (2019). ¿Es esto Educación Física?: el tiempo de compromiso motor en Educación Primaria. Sportis Sci J, 5 (3), 373-391. 